Open Access

\title{
Reduced ratio of eicosapentaenoic acid and docosahexaenoic acid to arachidonic acid is associated with early onset of acute coronary syndrome
}

\author{
Shusuke Yagi ${ }^{1 *}$, Ken-ichi Aihara², Daiju Fukuda ${ }^{1}$, Akira Takashima', Mika Bando', Tomoya Hara', \\ Sachiko Nishimoto ${ }^{3}$, Takayuki Ise ${ }^{1}$, Kenya Kusunose ${ }^{1}$, Koji Yamaguchi $^{1}$, Takeshi Tobiume ${ }^{1}$, Takashi Iwase ${ }^{1}$, \\ Hirotsugu Yamada ${ }^{1}$, Takeshi Soeki ${ }^{1}$, Tetsuzo Wakatsuki ${ }^{1}$, Michio Shimabukuro ${ }^{4}$, Masashi Akaike ${ }^{5}$ and Masataka Sata ${ }^{1}$
}

\begin{abstract}
Background: The hospitalization rate for acute coronary syndrome (ACS) for people aged $\leq 50$ has remained stable over the past decade. Increased serum levels of $n-3$ polyunsaturated fatty acids (PUFAs), such as eicosapentaenoic acid (EPA) and docosahexaenoic acid (DHA), are associated with a decreased incidence of cardiovascular events and mortality in older patients; however, it is currently unknown whether reduced serum levels of n-3 PUFAs is also a risk factor for ACS in patients aged $\leq 50$ years.

Methods and results: We retrospectively reviewed 102 (male/ female 73/29) Japanese ACS patients whose serum levels of EPA/arachidonic acid (AA) and DHA/AA were evaluated on admission. The EPA/AA ratio was the lowest in patients aged $\leq 50$ compared to patients aged $51-74$ and $\geq 75$. Pearson correlation analysis showed that early ACS onset was associated with Iow EPA/AA and DHA/AA ratios, and multiple regression analysis determined that decreased ratios of EPA/AA and DHA/AA, and male sex, current smoker status, increased body mass index and triglyceride levels, independently correlated with early ACS onset. Conversely, low-density and high-density lipoproteins, glycated hemoglobin, and hypertension did not correlate with early ACS onset. Subgroup analyses of male patients revealed that decreased ratios of EPA/AA and DHA/AA independently correlated with early ACS onset.

Conclusion: Decreased EPA/AA and DHA/AA ratios may be risk factors for early onset of ACS, suggesting that reduced EPA/AA and DHA/AA may represent targets for preventing ACS in Japanese young people.
\end{abstract}

Keywords: Polyunsaturated fatty acids (PUFA), Eicosapentaenoic acid (EPA), Docosahexaenoic acid (DHA), Acute coronary syndrome (ACS)

\section{Background}

In relation to the increased numbers of elderly people, the rate of young patients with acute coronary syndrome (ACS), including acute myocardial infarction (AMI), in Japan has been relatively decreasing; however, the age-

* Correspondence: syagi@tokushima-u.ac.jp

${ }^{1}$ Department of Cardiovascular Medicine, Institute of Biomedical Sciences, Tokushima University Graduate School, 3-18-15 Kuramoto, Tokushima 770-8503, Japan

Full list of author information is available at the end of the article adjusted incidence of AMI over the last 30 years has conversely been increasing, especially in men [1]. In addition, the clinical features and outcomes of ACS in young subjects ( $\leq 45$ years) are considered to differ from those in non-young subjects ( $>45$ years), owing to differences in the lifestyle and diet patterns [2]. Therefore, identification of new life-style related risk factors for ACS is important, especially in young subjects. It has been reported that reduced serum levels of $n-3$ polyunsaturated fatty acids (PUFAs), including eicosapentaenoic acid (EPA) and docosahexaenoic acid (DHA), are associated with an increased incidence of cardiovascular 
events and mortality [3, 4]. Additionally, a decreased ratio of serum EPA/arachidonic acid (AA) has been demonstrated to significantly correlate with the onset of ACS [5], and the serum EPA/AA ratio has been shown to stepwise increase with age [6], suggesting that reduced serum levels of n-3 PUFAs may represent a risk factor for ACS, especially in young subjects. However, it is currently unknown whether reduced serum levels of n-3 PUFAs is a risk factor for early onset ACS, and, accordingly, this study aimed to investigate this issue.

\section{Material and methods}

\section{Patients and study design}

We retrospectively reviewed 102 consecutive Japanese patients diagnosed with ACS who underwent emergent percutaneous coronary intervention in the Department of Cardiovascular Medicine at Tokushima University Hospital between January 2009 and June 2014. The patients were stratified into 3 groups according to age $(\leq 50,51-74$, and $\geq 75$ years $)$ and analyzed.

Because all patients aged $\leq 50$ were male, we moreover performed subgroup analyses after excluding all female patients, resulting in a total of 73 male patients out of the total 102 ACS patients being included in the analyses.

ACS included AMI and unstable angina. AMI was defined as a transient increase of the MB fraction of creatine kinase to a threshold of 3 times the 99th percentile of the upper reference limit (150 U/ L) after percutaneous coronary intervention in patients with ischemic symptoms and/or typical electrocardiographic findings (ST elevation) [7]. Unstable angina was defined as angina at rest, accelerated exertional angina combined with typical electrocardiographic changes (ST depression), or an increase in the intensity of anti-ischemic therapy with a transient increase of the MB fraction of creatine kinase to a threshold of less than 3 times the 99th percentile of the upper reference limit, as described previously [5]. The exclusion criteria were as follows: use of fish oil supplements or $\mathrm{n}-3$ fatty acid-containing drugs. In addition, patients with symptomatic, active malignant diseases or liver dysfunction (aspartate aminotransferase levels $>100 \mathrm{IU} / \mathrm{L}$, alanine aminotransferase levels $>100 \mathrm{IU} / \mathrm{L}$ ) were also excluded.

Hypertensive patients were defined as those with a systolic blood pressure $\geq 140 \mathrm{mmHg}$ and/or diastolic blood pressure $\geq 90 \mathrm{mmHg}$, and/or individuals receiving antihypertensive medications. Dyslipidemic patients were defined as those with a low-density lipoprotein cholesterol (LDL-C) level $\geq 140 \mathrm{mg} / \mathrm{dL}$, a triglyceride level $\geq 150 \mathrm{mg} /$ $\mathrm{dL}$, a high-density lipoprotein cholesterol (HDL-C) level $<40 \mathrm{mg} / \mathrm{dL}$, or individuals receiving lipid-lowering medications. Diabetic patients were defined as individuals receiving insulin or oral hypoglycemic agents or those with a glycated hemoglobin (HbA1c) level $\geq 6.5 \%$, fasting plasma glucose level $\geq 126 \mathrm{mg} / \mathrm{dL}$, or non-fasting plasma glucose level $\geq 200 \mathrm{mg} / \mathrm{dL}$.

Blood samples were drawn before emergent percutaneous coronary intervention on admission. The serum samples were stored at $-30{ }^{\circ} \mathrm{C}$ until assayed. The serum PUFAs composition, including the levels of EPA, DHA, and AA, was measured by gas-liquid chromatography at a commercially available laboratory (SRL, Tokyo, Japan) $[8,9]$. The intra- and inter-assay coefficients of variation for the EPA, DHA, and AA measurements were 1.3 and $3.3 \%, 1.5$ and $2.2 \%$, and 1.1 and $2.2 \%$, respectively. Since n-6 PUFAs, including AA, are considered proinflammatory fatty acids, and since the EPA/AA ratio is known to be associated with the incidence of cardiac events, the EPA/AA and DHA/AA ratios were calculated [5]. In addition, other biochemical parameters, including LDL-C, HDL-C, triglycerides, and HbA1c, were also measured. Body mass index was calculated as an index of obesity. The smoking status of the patient was obtained by interviews.

Written informed consent was not required because of the retrospective nature of the investigation. This study protocol was approved by the Tokushima University Hospital Ethics Committee and was conducted in accordance with the Declaration of Helsinki.

\section{Statistical analyses}

The Shapiro-Wilk test was performed to evaluate whether or not the parameters were normally distributed. Nonnormally distributed parameters were expressed as median and quartiles. Categorical parameters are expressed as numbers and percentages. After stratification according to age, differences in the clinical characteristics were determined by one-way ANOVA or Chi-square tests. Pearson correlation analysis was performed to determine the association between the age at ACS onset and the EPA/AA or DHA/AA ratio. Multiple regression analysis was used to assess the degrees of association among the variables, with age at ACS onset as the outcome variable. Age, body mass index, and the triglyceride, HDL-C, LDLC, HbA1c, EPA, DHA, AA, EPA/AA, and DHA/AA levels were natural $\log$ transformed for the statistical analyses, because of their non-normal distributions. All statistical analyses were performed using JMP 10 software (SAS, Cary, NC, USA). Statistical significance was defined as $P<0.05$.

\section{Results}

\section{Clinical characteristics of subjects}

The characteristics of all patients stratified by age are shown in Table 1. There were significant differences in the EPA/AA ratios among the groups. In addition, there were significant differences in the male sex ratio, body 
Table 1 Clinical characteristics of all patients stratified by age

\begin{tabular}{|c|c|c|c|c|c|}
\hline Variables & Total & $35-50 y$ & $51-74$ y & $\geq 75 y$ & $P$-value \\
\hline Number of patients & 102 & 11 & 57 & 34 & - \\
\hline Male, n (\%) & $73(71.6 \%)$ & $11(100 \%)$ & $46(80.7 \%)$ & $16(47.1 \%)$ & $<0.001$ \\
\hline Age (years) & $69(56-77)$ & $42(38-49)$ & $64(56-70)$ & $79(77-84)$ & $<0.001$ \\
\hline Body mass index $\left(\mathrm{kg} / \mathrm{m}^{2}\right)$ & $24(22-25)$ & $26(25-29)^{*},+\dagger$ & $24(21-26)$ & $22(20-24)$ & $<0.01$ \\
\hline Triglycerides (mg/dL) & $111(67-171)$ & $143(132-194)$ & $110(63-177)$ & $95(66-129)$ & 0.09 \\
\hline $\mathrm{HDL}-\mathrm{C}(\mathrm{mg} / \mathrm{dL})$ & 45 (39-59) & $42(34-43)^{*}$ & $47(40-60)$ & $45(38-60)$ & 0.04 \\
\hline LDL-C (mg/dL) & $114(92-137)$ & $133(101-185)$ & $113(90-132)$ & $117(84-148)$ & 0.09 \\
\hline HbA1c (\%) & $5.9(5.6-6.4)$ & $5.8(5.4-6.7)$ & $6.0(5.5-6.6)$ & $5.9(5.6-6.4)$ & 0.53 \\
\hline \multicolumn{6}{|l|}{ Fatty acid concentrations } \\
\hline $\mathrm{EPA}(\mu \mathrm{g} / \mathrm{mL})$ & $43(29-65)$ & $28(21-55)^{*}, \dagger$ & $43(34-65)$ & $46(30-69)$ & 0.12 \\
\hline $\mathrm{DHA}(\mu \mathrm{g} / \mathrm{mL})$ & 119 (97-149) & $102(94-140)$ & $118(97-150)$ & $125(97-163)$ & 0.36 \\
\hline $\mathrm{AA}(\mu \mathrm{g} / \mathrm{mL})$ & $165(141-206)$ & $193(164-234)$ & $167(152-203)$ & $143(126-211)$ & 0.09 \\
\hline EPA/AA & $0.25(0.17-0.37)$ & $0.17(0.07-0.23)^{*}, \dagger$ & $0.26(0.18-0.37)$ & $0.29(0.19-0.42)$ & 0.03 \\
\hline DHA/AA & $0.75(0.59-0.96)$ & $0.70(0.47-0.89)$ & $0.67(0.56-0.95)$ & $0.83(0.70-1.00)$ & 0.05 \\
\hline \multicolumn{6}{|l|}{ Complications } \\
\hline Dyslipidemia, n (\%) & $38(37 \%)$ & $6(55 \%)$ & $22(39 \%)$ & $10(29 \%)$ & 0.31 \\
\hline Hypertension, n (\%) & $63(62 \%)$ & $5(45 \%)$ & $34(60 \%)$ & $24(71 \%)$ & 0.29 \\
\hline Diabetes mellitus, n (\%) & $29(28 \%)$ & $2(18 \%)$ & 19 (33 \%) & $8(24 \%)$ & 0.44 \\
\hline Current smoking, n (\%) & $44(43 \%)$ & $9(82 \%)$ & $28(49 \%)$ & 7 (21\%) & $<0.01$ \\
\hline \multicolumn{6}{|l|}{ Drugs } \\
\hline ACEI/ARB, n (\%) & $27(26 \%)$ & $1(9 \%)$ & $11(19 \%)$ & $15(44 \%)$ & 0.01 \\
\hline$\beta$-blockers, n (\%) & $10(10 \%)$ & 1 (9\%) & $4(7 \%)$ & $5(15 \%)$ & 0.49 \\
\hline Calcium channel blockers, n (\%) & $27(26 \%)$ & $1(9 \%)$ & $15(26 \%)$ & $11(32 \%)$ & 0.31 \\
\hline Statins, n (\%) & $15(15 \%)$ & $2(18 \%)$ & $11(19 \%)$ & $2(6 \%)$ & 0.20 \\
\hline Aspirin, n (\%) & 16 (16 \%) & 2 (18\%) & $8(14 \%)$ & $6(18 \%)$ & 0.28 \\
\hline
\end{tabular}

Unless indicated otherwise, data are presented as median and quartiles

Abbreviations: $A A$ arachidonic acid, $A C E I$ angiotensin converting enzyme inhibitors, $A R B$ angiotensin II receptor blockers, DHA docosahexaenoic acid, EPA eicosapentaenoic acid, $\mathrm{HbA1c}$ glycated hemoglobin, $\mathrm{HDL}-\mathrm{C}$ high-density lipoprotein cholesterol, $L D L-C$ low-density lipoprotein cholesterol

* $P<0.05$ (vs. age $51-74$ )

$+P<0.05$ (vs. age $\geq 75$ )

$+\dagger P<0.01$ (vs. age $\geq 75$ )

mass index, and HDL-C levels, prevalence of current smokers, and prevalence of angiotensin-converting enzyme inhibitors/angiotensin II receptor blocker use, but not in the triglyceride, LDL-C, HbA1c, EPA, DHA, and AA levels, DHA/AA ratio, prevalence of dyslipidemia, hypertension, and diabetes mellitus, and the other administered drugs. Pearson correlation analysis showed that the levels of EPA and DHA were positively associated with the EPA/AA and DHA/AA ratios, respectively, in all patients (Additional file 1: Figure S1).

Of note, the patients aged 35-50 were all men. Therefore, we moreover performed subgroup analyses of male patients stratified by age (Additional file 2: Table S1). A significant difference was observed for the EPA/AA ratio, but not DHA/AA.

The female patient characteristics stratified by age are shown (Additional file 2: Table S2).

\section{Correlation between n-3 PUFAs and age at ACS onset}

The Pearson correlation analysis showed that age at ACS onset was positively associated with the EPA/AA and DHA/AA ratios in all (Fig. 1) and male patients (Additional file 1: Figure S2).

Furthermore, multiple regression analysis in all patients revealed that male sex, and body mass index, prevalence of current smoker, and level of triglycerides were negative, and levels of EPA/AA or DHA/AA were positive contributors to the age at ACS onset (Table 2, Model 1, 2). However, levels of LDL-C, HDL-C, HbA1c, and prevalence of hypertension were statistically excluded. Multiple regression analysis in male patients revealed that levels of EPA/AA or DHA/AA were positive contributors and level of triglycerides was a negative contributor to the age at ACS onset. However, the body mass index, levels of LDL-C, HDL-C, and HbA1c, 

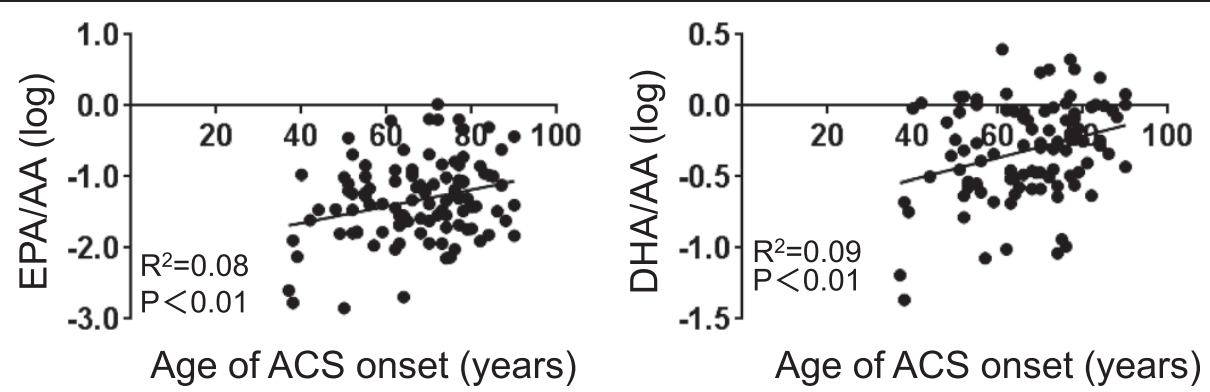

Fig. 1 Pearson correlation analyses in all patients between the age at ACS onset and levels of EPA/AA and DHA/AA. AA arachidonic acid, ACS acute coronary syndrome, DHA docosahexaenoic acid, EPA eicosapentaenoic acid

prevalence of hypertension and current smoker status were statistically excluded (Additional file 2: Table S3, Model 1, 2).

\section{Discussion}

Herein, we showed that the EPA/AA ratio was the lowest in early-onset ACS patients and that low levels of EPA/AA and DHA/AA were independent contributors of early onset of ACS.

Table 2 Multiple regression analysis for determinants of the age of acute coronary syndrome onset

\begin{tabular}{llll}
\hline Variables & Coefficient & $95 \% \mathrm{Cl}$ & $P$-value \\
\hline Model 1 & & & \\
Male sex & -0.06 & -0.10 to -0.02 & $<0.01$ \\
Body mass index & -0.27 & -0.50 to -0.05 & 0.02 \\
Hypertension & 0.04 & -0.001 to 0.07 & 0.05 \\
Current smoker & -0.04 & -0.08 to 0.001 & 0.06 \\
LDL-C & -0.04 & -0.12 to 0.04 & 0.28 \\
Triglycerides & -0.08 & -0.14 to -0.02 & 0.01 \\
HDL-C & 0.01 & -0.13 to 0.16 & 0.85 \\
HbA1C, \% & 0.20 & -0.03 to 0.44 & 0.09 \\
EPA/AA & 0.07 & 0.01 to 0.13 & 0.02
\end{tabular}

Model 2

\begin{tabular}{llll} 
Male sex & -0.06 & -0.10 to -0.02 & $<0.01$ \\
Body mass index & -0.24 & -0.46 to -0.01 & 0.04 \\
Hypertension & 0.03 & -0.001 to 0.07 & 0.05 \\
Current smoker & -0.04 & -0.08 to 0.01 & 0.04 \\
LDL-C & -0.04 & -0.11 to 0.04 & 0.31 \\
Triglycerides & -0.09 & -0.15 to -0.03 & $<0.01$ \\
HDL-C & 0.05 & -0.10 to 0.19 & 0.53 \\
HbA1C, \% & 0.18 & -0.05 to 0.41 & 0.12 \\
DHA/AA & 0.15 & -0.05 to 0.25 & $<0.01$ \\
\hline
\end{tabular}

Model 1, $\mathrm{R}^{2}=0.37 ; P<0.001$

Model 2, $\mathrm{R}^{2}=0.39 ; P<0.001$

Abbreviations: $A A$ arachidonic acid, $C l$ confidence interval, $D H A$

docosahexaenoic acid; EPA eicosapentaenoic acid, $H b A 1 c$ glycated hemoglobin, $H D L-C$ high-density lipoprotein cholesterol, $L D L-C$ low-density lipoprotein cholesterol
Although patients with CAD typically become symptomatic after age 40, autopsy studies have demonstrated that coronary atherosclerosis can begins as early as 20 years of age [10-13], and such atherosclerotic changes in the coronary arteries have also been observed in Japanese youths, whose incidence and mortality from CAD are reportedly the lowest in all industrialized countries [14].

Despite recent advances in the identification of risk factors, the occurrence of CAD has continued to increase worldwide, and despite the decrease in cardiovascular mortality due to statin treatment, two out of three patients still experience cardiovascular events [15]. In addition, the AMI hospitalization rate for relatively young people has remained stable over the past decade [16-18], which may be largely due to the insufficient control of the various CAD risk factors [1]. Several risk factors for early-onset AMI have been reported; classical risk factors include serum cholesterol levels, systolic blood pressure, and cigarette smoking [19], while nonclassical risk factors include vasospastic tendencies, thrombophilic conditions, and a history of Kawasaki disease $[20,21]$. However, there are currently no universal guidelines on the prevention of CAD in young people, in part because of insufficient evidence on coronary disease prevention in adults younger than 40 years [19]. Therefore, sufficient control of classical CAD risk factors and identification of residual risk factors for CAD are needed.

It has been reported that reduced serum levels of $n-3$ PUFA, especially EPA, are associated with an increased incidence of cardiovascular events and mortality [3-5], and the JELIS study, a large clinical trial, showed effects of EPA on reducing cardiovascular events in hypercholesterolemic patients [22]. However, it should be noted that the levels of EPA $(95 \mu \mathrm{g} / \mathrm{mL})$ and DHA $(170 \mu \mathrm{g} /$ $\mathrm{mL}$ ) in the hyperlipidemic patients without ACS enrolled in the JELIS trial were higher than those in the ACS patients in our study (EPA, $43 \mu \mathrm{g} / \mathrm{mL}$; DHA, $119 \mu \mathrm{g} / \mathrm{mL}$ ) [23]. Furthermore, the Japanese National Health and Nutrition Survey demonstrated that the type of nutrition 
has changed from Japanese traditional style to western style; in particular, fish intake has been decreasing, especially in people aged $30-49$ and $40-49$ years (http://www0.nih.go.jp/eiken/english/research/program epidemiology.html), suggesting that low levels of n-3 PUFAs could lead to CAD prevention in young people.

In the study, all patients aged 50 or under were men. It has been well established that male gender is a risk factor of CAD [24, 25], and previous studies have shown that men with ACS are more likely to have ruptured plaques, whereas women are more likely to present with plaque erosion, suggesting that the mechanism of ACS development in men and women differs [24]. For example, it has been speculated that estrogen might contribute to delaying the plaque development, stabilizing the existing plaques, and preventing plaque rupture in women [25].

On the other hand, the underlying mechanisms responsible for the favorable effects of n-3 PUFAs remain partly unknown. n-3 PUFAs have been demonstrated to induce stability of vulnerable plaques, which is considered to contribute to the protection against acute cardiovascular events, and it has been shown that administration of fish oil increased the proportions of n-3 PUFAs in carotid plaque lipids and increased the proportions of well-formed fibrous caps, rather than thin inflamed caps, as compared with a placebo or sunflower oil [26]. In addition, a recent study showed that administration of EPA increased the fibrous cap thickness of coronary atherosclerotic plaques [27].

Moreover, several studies have shown that long-chain n-3 PUFAs can decrease the expressions of intercellular adhesion molecule- 1 and vascular cell adhesion molecule1 on the surface of endothelial cells [28] and monocytes [29]. Therefore, n-3 PUFA-induced decreases in adhesion molecule expression on endothelial cells and/or the monocyte/macrophage itself, might reduce migration of monocytes/macrophages into the plaque [30,31]. Further, chylomicron remnant-like particles enriched in n-3 PUFA are taken up more slowly by macrophages than those enriched in saturated or monounsaturated fatty acids, which might also be involved in attenuation of plaque formation [32], and it has been shown that administration of $\mathrm{n}-3$ PUFA reduced the matrix metalloproteinase- $7,-9$ and -12 expressions [33]. These gene products are proteases capable of degrading extracellular matrix proteins, consequently leading to plaque rupture [33]. Accordingly, reduced expression of matrix metalloproteinases by $n-3$ PUFAs may also contribute to plaque instability, leading to suppression of ACS.

Although some meta-analyses and clinical trials have shown beneficial effects of $n-3$ PUFAs on cardiovascular events [22, 34], a previous systematic review conversely showed no definite effects of n-3 PUFAs on cardiovascular events [35]. In addition, some theoretical unfavorable effects have been reported, including one study showing that intake of dietary PUFAs such as EPA and DHA increased lipid peroxidation, which may in turn enhance oxidative stress and cancel the preferable effects of $n-3$ PUFAs [36]. This unfavorable effect of n-3 PUFAs may explain the inconsistent clinical results and contribute to the finding that the characteristics of patients who received beneficial effects of PUFAs differ among studies [37, 38].

The present study had several limitations. This was a retrospective study-with a relatively small sample size-that was performed in a single-center located in a costal provincial city. Larger clinical cohort studies are needed to confirm our results and to clarify the effects of n-3 PUFAs on early-onset ACS.

\section{Conclusions}

We here demonstrated that decreased EPA/AA and DHA/AA ratios may be risk factors for early onset of ACS, suggesting that reduced EPA/AA and DHA/AA may represent targets for preventing ACS in Japanese young people.

\section{Additional files}

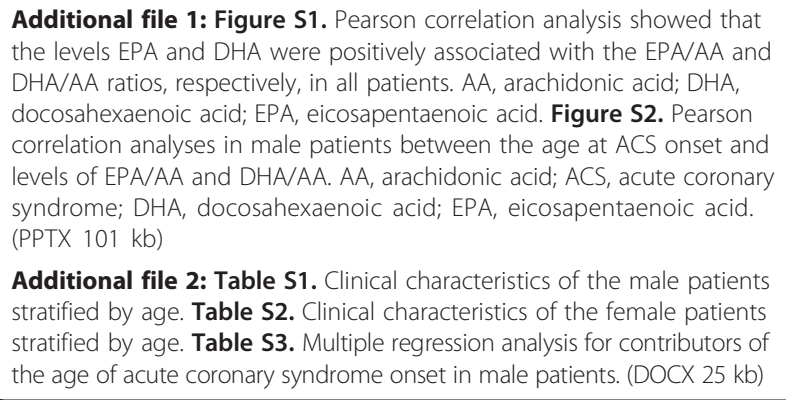

Additional file 2: Table S1. Clinical characteristics of the male patients stratified by age. Table S2. Clinical characteristics of the female patients stratified by age. Table S3. Multiple regression analysis for contributors of the age of acute coronary syndrome onset in male patients. (DOCX $25 \mathrm{~kb}$ )

\section{Abbreviations}

ACS: acute coronary syndrome; AMI: acute myocardial infarction; CAD: coronary artery disease; PUFAs: polyunsaturated fatty acids; EPA: eicosapentaenoic acid; DHA: docosahexaenoic acid; AA: arachidonic acid; LDL-C: low-density lipoprotein cholesterol; HDL-C: high-density lipoprotein cholesterol; HbA1c: glycated hemoglobin.

\section{Competing interests}

The authors declare that they have no competing interests.

\section{Authors' contributions}

SY, KA, DF, AT, MB, TH, SN, TIS, KK, KY, TT, TIW, HY, TS, TW, collected data and SY analyzed the data and wrote the manuscript. MSh, MA, MSa supervised the study. All authors read and approved the final manuscript.

\section{Acknowledgements}

We thank the staff of the Hospital Information Center of Tokushima

University Hospital for retrieving the patient data from the medical records. This work was supported in part by JSPS KAKENHI (Grant Numbers 25461131 and 25860605). 


\section{Author details}

${ }^{1}$ Department of Cardiovascular Medicine, Institute of Biomedical Sciences, Tokushima University Graduate School, 3-18-15 Kuramoto, Tokushima 770-8503, Japan. ²Department of Hematology, Endocrinology and Metabolism, Institute of Biomedical Sciences, Tokushima University Graduate School, 3-18-15 Kuramoto, Tokushima 770-8503, Japan. ${ }^{3}$ Department of Nutrition and Metabolism, Institute of Biomedical Sciences, Tokushima University Graduate School, 3-18-15 Kuramoto, Tokushima 770-8503, Japan. ${ }^{4}$ Department of Cardio-Diabetes Medicine, Institute of Biomedical Sciences, Tokushima University Graduate School, 3-18-15 Kuramoto, Tokushima 770-8503, Japan. ${ }^{5}$ Department of Medical Education, Institute of Biomedical Sciences, Tokushima University Graduate School, 3-18-15 Kuramoto, Tokushima 770-8503, Japan.

Received: 15 June 2015 Accepted: 12 October 2015 Published online: 29 October 2015

\section{References}

1. Takii T, Yasuda S, Takahashi J, Ito K, Shiba N, Shirato K, et al. Trends in acute myocardial infarction incidence and mortality over 30 years in Japan: report from the MIYAGI-AMI Registry Study. Circ J. 2010;74:93-100.

2. Jinnouchi H, Sakakura K, Wada H, Kubo N, Sugawara Y, Funayama H, et al. Clinical features of myocardial infarction in young Japanese patients. Int Heart J. 2013:54:123-8.

3. Amano T, Matsubara T, Uetani T, Kato M, Kato B, Yoshida T, et al. Impact of omega-3 polyunsaturated fatty acids on coronary plaque instability: an integrated backscatter intravascular ultrasound study. Atherosclerosis. 2011;218:110-6.

4. Hara M, Sakata Y, Nakatani D, Suna S, Usami M, Matsumoto S, et al. Low levels of serum n-3 polyunsaturated fatty acids are associated with worse heart failure-free survival in patients after acute myocardial infarction. Circ J. 2013;77:153-62.

5. Nishizaki Y, Shimada K, Tani S, Ogawa T, Ando J, Takahashi M, et al. Significance of imbalance in the ratio of serum n-3 to $n-6$ polyunsaturated fatty acids in patients with acute coronary syndrome. Am J Cardiol. 2014;113:441-5.

6. Yanagisawa N, Shimada K, Miyazaki T, Kume A, Kitamura Y, Ichikawa R, et al. Polyunsaturated fatty acid levels of serum and red blood cells in apparently healthy Japanese subjects living in an urban area. J Atheroscler Thromb. 2010;17:285-94.

7. Thygesen K, Alpert JS, Jaffe AS, Simoons ML, Chaitman BR, White HD, et al. Third universal definition of myocardial infarction. Circulation. 2012;126:2020-35.

8. Yagi S, Aihara Kl, Fukuda D, Takashima A, Hara T, Hotchi J, et al. Effects of docosahexaenoic acid on the endothelial function in patients with coronary artery disease. J Atheroscler Thromb. 2014.

9. Yagi S, Hara T, Ueno R, Aihara Kl, Fukuda D, Takashima A, et al. Serum concentration of eicosapentaenoic acid is associated with cognitive function in patients with coronary artery disease. Nutr J. 2014;13:112.

10. McNamara JJ, Molot MA, Stremple JF, Cutting RT. Coronary artery disease in combat casualties in Vietnam. JAMA. 1971:216:1185-7.

11. Tuzcu EM, Kapadia SR, Tutar E, Ziada KM, Hobbs RE, McCarthy PM, et al. High prevalence of coronary atherosclerosis in asymptomatic teenagers and young adults: evidence from intravascular ultrasound. Circulation. 2001;103:2705-10.

12. McGill Jr HC, McMahan CA, Zieske AW, Tracy RE, Malcom GT, Herderick EE, et al. Association of Coronary Heart Disease Risk Factors with microscopic qualities of coronary atherosclerosis in youth. Circulation. 2000;102:374-9.

13. Kisanuki A, Asada Y, Sato Y, Marutsuka K, Takeda K, Sumiyoshi A. Coronary atherosclerosis in youths in Kyushu Island, Japan: histological findings and stenosis. J Atheroscler Thromb. 2000:6:55-9.

14. Ueshima H, Sekikawa A, Miura K, Turin TC, Takashima N, Kita Y, et al. Cardiovascular disease and risk factors in Asia a selected review. Circulation. 2008;118:2702-9.

15. Libby $P$. The forgotten majority: unfinished business in cardiovascular risk reduction. J Am Coll Cardiol. 2005:46:1225-8.

16. Gupta A, Wang Y, Spertus JA, Geda M, Lorenze N, Nkonde-Price C, et al. Trends in acute myocardial infarction in young patients and differences by sex and race, 2001 to 2010. J Am Coll Cardiol. 2014;64:337-45.

17. Rumana N, Kita Y, Turin TC, Murakami Y, Sugihara H, Morita Y, et al. Trend of increase in the incidence of acute myocardial infarction in a Japanese population: Takashima AMI Registry, 1990-2001. Am J Epidemiol. 2008; 167:1358-64

18. Kitamura A, Sato S, Kiyama M, Imano H, Iso H, Okada T, et al. Trends in the incidence of coronary heart disease and stroke and their risk factors in Japan, 1964 to 2003 - The Akita-Osaka study. J Am Coll Cardiol. 2008;52:71-9.

19. Navas-Nacher EL, Colangelo L, Beam C, Greenland P. Risk factors for coronary heart disease in men 18 to 39 years of age. Ann Intern Med. 2001;134:433-9.

20. Doughty M, Mehta R, Bruckman D, Das S, Karavite D, Tsai T, et al. Acute myocardial infarction in the young-The University of Michigan experience. Am Heart J. 2002;143:56-62.

21. Yagi S, Tsuda E, Shimizu W, Kurita T, Seguchi O, Nonogi H, et al. Two adults requiring implantable defibrillators because of ventricular tachycardia and left ventricular dysfunction caused by presumed Kawasaki disease. Circ J. 2005;69:870-4

22. Yokoyama M, Origasa H, Matsuzaki M, Matsuzawa Y, Saito Y, Ishikawa Y, et al. Effects of eicosapentaenoic acid on major coronary events in hypercholesterolaemic patients (JELIS): a randomised open-label, blinded endpoint analysis. Lancet. 2007;369:1090-8.

23. Itakura H, Yokoyama M, Matsuzaki M, Saito Y, Origasa H, Ishikawa Y, et al. Relationships between plasma fatty acid composition and coronary artery disease. J Atheroscler Thromb. 2011;18:99-107.

24. Hochman JS, Tamis JE, Thompson TD, Weaver WD, White HD, Van de Werf $\mathrm{F}$, et al. Sex, clinical presentation, and outcome in patients with acute coronary syndromes. N Engl J Med. 1999;341:226-32.

25. Burke AP, Farb A, Malcom G, Virmani R. Effect of menopause on plaque morphologic characteristics in coronary atherosclerosis. Am Heart J. 2001;141:S58-62

26. Thies F, Garry JMC, Yaqoob P, Rerkasem K, Williams J, Shearman CP, et al. Association of $n-3$ polyunsaturated fatty acids with stability of atherosclerotic plaques: a randomised controlled trial. Lancet. 2003;361:477-85.

27. Yamano T, Kubo T, Shiono Y, Shimamura K, Orii M, Tanimoto T, et al. Impact of eicosapentaenoic acid treatment on the fibrous cap thickness in patients with coronary atherosclerotic plaque: an optical coherence tomography study. J Atheroscler Thromb. 2014.

28. De Caterina R, Cybulsky Ml, Clinton SK, Gimbrone Jr MA, Libby P. The omega-3 fatty acid docosahexaenoate reduces cytokine-induced expression of proatherogenic and proinflammatory proteins in human endothelial cells. Arterioscler Thromb. 1994;14:1829-36.

29. Hughes DA, Southon S, Pinder AC. (n-3) Polyunsaturated fatty acids modulate the expression of functionally associated molecules on human monocytes in vitro. J Nutr. 1996;126:603-10.

30. Kris-Etherton PM, Harris WS, Appel $\amalg$, American Heart Association. Nutrition C: Fish consumption, fish oil, omega-3 fatty acids, and cardiovascular disease. Circulation. 2002;106:2747-57.

31. Calder PC. Long-chain n-3 fatty acids and cardiovascular disease: further evidence and insights. Nutr Res. 2004;24:761-72.

32. De Pascale C, Avella M, Perona JS, Ruiz-Gutierrez V, Wheeler-Jones CPD, Botham KM. Fatty acid composition of chylomicron remnant-like particles influences their uptake and induction of lipid accumulation in macrophages. FEBS J. 2006;273:5632-40

33. Cawood AL, Ding R, Napper FL, Young RH, Williams JA, Ward MJA, et al. Eicosapentaenoic acid (EPA) from highly concentrated $n-3$ fatty acid ethyl esters is incorporated into advanced atherosclerotic plaques and higher plaque EPA is associated with decreased plaque inflammation and increased stability. Atherosclerosis. 2010;212:252-9.

34. Bucher HC, Hengstler P, Schindler C, Meier G. N-3 polyunsaturated fatty acids in coronary heart disease: a meta-analysis of randomized controlled trials. Am J Med. 2002;112:298-304.

35. Hooper L, Thompson RL, Harrison RA, Summerbell CD, Ness AR, Moore HJ, et al. Risks and benefits of omega 3 fats for mortality, cardiovascular disease, and cancer: systematic review. BMJ. 2006;332:752-60.

36. Allard JP, Kurian R, Aghdassi E, Muggli R, Royall D. Lipid peroxidation during n3 fatty acid and vitamin E supplementation in humans. Lipids. 1997;32:535-41.

37. Kromhout D, Yasuda S, Geleijnse JM, Shimokawa H. Fish oil and omega-3 fatty acids in cardiovascular disease: do they really work? Eur Heart J. 2012;33:436-43.

38. Marklund M, Leander K, Vikstrom M, Laguzzi F, Gigante B, Sjogren $P$, et al. Polyunsaturated fat intake estimated by circulating biomarkers and risk of cardiovascular disease and all-cause mortality in a population-based cohort of 60-year-old men and women. Circulation. 2015;132(7):586-94. 